\title{
Development and Application of the Special Tools for the Cleaning of Screw Fittings
}

\author{
Zhang $\mathrm{Su}^{1, \mathrm{a}}$, Zhu Yuanda ${ }^{1, \mathrm{a}}$, Dai Dongxu ${ }^{2, \mathrm{~b}}$, Zheng Wanyi ${ }^{1, \mathrm{a}}$, Han Zhe $^{3, \mathrm{c}}$ \\ ${ }^{1}$ Liaoning Skill Training Center \\ 2 Benxi Power Supply Company, State Grid Liaoning Electric Power Supply Co., Ltd. \\ ${ }^{3}$ Anshan Power Supply Company, State Grid Liaoning Electric Power Supply Co., Ltd. \\ a22181221@qq.com, b bxwl_650928@126.com, ${ }^{\mathrm{C}}$ 1422688@qq.com,
}

Keywords: isolating switch, cleaning

\begin{abstract}
The isolating switch is a device of isolating voltage in order to ensure the safety of the maintenance work of high voltage apparatus and equipment. It is installed by bolts on the mounting rack of electric equipment. The isolating switch is exposed outdoors and always affected by wind, rain, dust and so on, resulting in corrosion, sludge attachment and irregular thread shape of the isolating switch bolt, which influences the smoothness of screwing isolating switch bolts, and causes inconvenient disassembly and assembly. Therefore, it is necessary to regularly clean, and coat them with grease. The current cleaning method of isolating switch bolts is to disassemble all isolating switch bolts, put them into the box or the bucket with cleaner, and brush the isolating switch bolts manually. There is only one containing space inside the box or bucket, so the bolts of different models inside are mixed and disorderly, and therefore the sorting time is long. Besides, operators need to put hands into the cleaner while stirring and cleaning the isolating switch bolts, which is troublesome and easy to damage the operators' arms. In order to solve the above technical problems, this paper provides a cleaning tank of isolating switch bolts with orderly screw placement, shorter cleaning operation time, and easy and safe operation.
\end{abstract}

\section{Introduction}

This paper is aimed at a kind of cleaning tank of isolating switch bolts whose special places are as follows: There is a seated tray in the tank body, the outer bottom of the tray is located above the inner bottom of the tank, the tray is equipped with a plurality of different sizes of cleaning grooves, the bottom of each cleaning tank is provided with a circulation hole, and the upper surface of the clapboard of both adjacent cleaning grooves is provided with a slot, where the labels of the model of the isolating switch bolts can be put, and therefore it is convenient to quickly find the bolt.

Various kinds of fouling harmful to production operation, product quality or human health are left, deposited and generated on their surface and become industrial fouling when equipment, pipelines, factories, buildings, raw materials, products, semi-products, etc. contact to air, environment, raw materials, media, products, mechanical oil, etc., due to the physical, chemical and electrochemical or biological function. Industrial fouling must be eliminated by technical measures, otherwise the appearance of pollutants is damaged, the material is eroded, production cannot be done normally, the product quality is reduced, raw materials and energy consumption increases, and production accidents occur frequently. The process of adopting physical, chemical or biological methods to remove the fouling regularly or irregularly is called industrial fouling cleaning.

\section{Classification}

The surface treatment of the bolts has the following types:

Electro galvanizing: Electro galvanizing technology is a traditional technology of metal coating treatment, which provides the basic corrosion resistance of the metal surface. Hot-dip galvanizing: Hot-dip galvanizing, with good resistance to corrosion and sacrificial protective effect to steel 
matrix, resistant to high weather and salt water erosion is suitable for chemical plants, refineries and coastal and offshore work platform.

Hot-dip aluminizing: Hot-dip aluminized steel has high strength of steel matrix and corrosion resistance, abrasion resistance, high temperature resistance and other characteristics of aluminum layer, and its corrosion resistance is 5-10 times that of galvanized steel. For sulfide and corrosion resistance, its performance is better than that of 18-8 stainless steel, that is, it can resist the high temperature of 600 degrees. It has obvious economic benefit.

Dacromet: Dacromet coating is even silver grey, $80 \%$ of which is thin zinc sheets and aluminum sheets, and the rest of which is the chromate. It has excellent performance, such as strong resistance to corrosion, 7-10 times better than electro galvanizing, salt spray test $>500$ hours, and no hydrogen embrittlement. It is especially suitable for high strength mechanical parts, such as the high strength bolt for subway construction. It has high heat resistance, which is stable at 300 degrees, also has high permeability, adhesion, reducing friction, weather stability, and resistance to chemical stability, and no environmental pollution and other advantages.

Cadmium plating: Cadmium plating is a positive grade coating, which is mainly used for technical anticorrosion. The main advantage is its good brazing property and suitable contact resistance. Owing to good lubrication, cadmium plating is often used in aviation, spaceflight, navigation, radio and electronic products. The coating has a double protective effect on the steel matrix, so the corrosion resistance is much better than that of zinc coating. But because of the high cost of cadmium plating (about 5 times as much as galvanizing) and the serious environment pollution, cadmium plating is not recommended, but normally replaced by galvanizing.

PTFE:PTFE coating, with significant anti-sticking property, is the most heat resistant material in all fluoride containing polymers, and nearly resists all the chemical substances. It has very low friction coefficient, excellent wear resistance and good chemical stability. The operating temperature of the PTFE coating can reach 300 degrees at most, and its applied thickness is usually 25-75 microns. Xylan:Xylan coating is a kind of fluorine containing polymer. Most of the Xylan coating contains PTFE and other lubricant composition, and it is generally used in the form of thin film to the surface treatment of a variety of metal components and fasteners. It has good ability of lubrication, wear resistance, corrosion resistance, friction resistance, heat resistance, and a unique property of non sticky, high chemical stability and no environmental pollution, etc..

\section{Design principle}

The isolating switch is a device of isolating voltage while ensuring the safety of the maintenance work of high voltage apparatus and equipment. It is installed by bolts on the mounting rack of electric equipment. The isolating switch is exposed outdoors and always affected by wind, rain, dust and so on, resulting in corrosion, sludge attachment and irregular thread shape of the isolating switch bolt, which influences the smoothness of screwing isolating switch bolts, and causes inconvenient disassembly and assembly. Therefore, it is necessary to regularly clean, and coat them with grease. The current cleaning method of isolating switch bolts is to disassemble all isolating switch bolts, put them into the box or the bucket with cleaner, and brush the isolating switch bolts manually. There is only one containing space inside the box or bucket, so the bolts of different models inside are mixed and disorderly, and therefore the sorting time is long. Besides, operators need to put hands into the cleaner while stirring and cleaning the isolating switch bolts, which is troublesome and easy to damage the operators' arms. In order to solve the above technical problems, this paper provides a cleaning tank of isolating switch bolts with orderly screw placement, shorter cleaning operation time, and easy and safe operation.

Water and non water solvent: the solvent of fouling is the material which can remove the fouling of the cleaning object in the form of dissolution or dispersion, and there is no new stable and chemical composed material is generated. It includes water and non water solvent.

(1) water: water is not only the existence of nature, but also the most important solvent. In industrial cleaning, water is not only the solvent of most chemical cleaning agents, but also the 
solvent of a lot of fouling. In the washing, when it can be removed by water will not use non water solvents and additives.

(2) non water solvent: non water solvent, including hydrocarbon, halogenated hydrocarbons, alcohols, ethers, ketones, esters, phenols and their mixtures, is mainly used in dissolving organic fouling, such as grease and some organic compounds fouling.

Surfactants: its molecules have both hydrophilic polar groups and oleophilic non polar groups. With only a small amount, it can greatly reduce the surface tension of the solvent (usually water) and the interfacial tension of the liquid. And it has the function of lubrication, solubilization, emulsification, dispersion and detergency.

Surfactants are classified in many ways and generally classified according to the ionization state of the solvent and the ionic type of the hydrophilic group. The most commonly used are anionic surfactants, cationic surfactants, ampholytic surfactants and nonionic surfactants, the first three kinds of which are ionic surfactants.

Surfactants are widely used in cleaning of the family life and industrial production.

Acid - alkali cleaning agent: By acid and alkali reaction (sometimes accompanied by oxidation reduction, etc.), the fouling can be dissolved or dispersed in the cleaning solution. Most are organic acids, inorganic acids, alkali and acid or alkaline salt.

Most of the acid - alkali cleaning agents are composed of acid, alkali, water solution and necessary auxiliary agent. Another type is the acid or alkali in melt state which takes effect with fouling in the condition of high temperature, so the fouling which is originally indissolvable or hard to dissolve in the cleaning medium, is transferred into soluble compounds. This kind of acid or alkali is commonly referred to as melting agents. The cleaning agent has good effect for the fouling which is difficult to remove by solvent or solution.

As the tray is seated in the tank and tray and tank are respectively set handles at both sides, which makes convenient transportation; the cleaning grooves of different sizes on the tray provide corresponding grooves for the different types of bolts, which makes the bolts placed in order, and avoid mixing of different types of bolts, and shorten the cleaning time. On the bottom plate of the tray is provided with circulation holes, so when the tray is put into or raised from the tank, the cleaning fluid in the tank would flow through the circulation hole automatically into or out of the cleaning tank. The operation is convenient; during cleaning operation, operators do not need to contact with the cleaning fluid, which increases the safety of operation. As shown in Fig. 1:

Brief Description

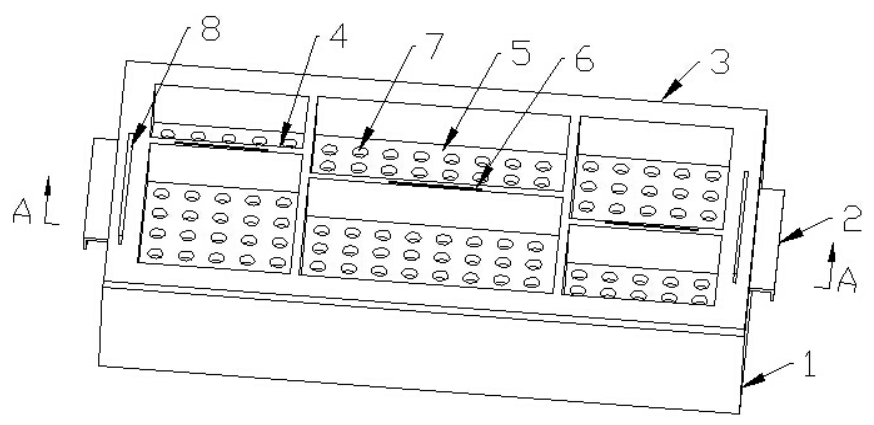

Figure1: Equipment structure

1- tank, 2- handle I, 3- tray, 4- clapboard, 5- cleaning groove, 6- slot, 7- circulation hole, 8- handle II

During operation, first of all, the appropriate amount of cleaning fluid is added into tank 1 , and then the different models of the isolating switch bolts are put into the corresponding cleaning groove 5 of tray 3 in order, and tray 3 is seated in tank 1, and at this time, the cleaning fluid flows through the circulation hole 7 into the cleaning groove 5 , when the isolating switch bolts of tank 5 are brushed. After cleaning, tray 3 is pulled out, the switch bolts is wiped by a cloth, and grease is applied, so the operation finishes. 


\section{Conclusion}

The isolating switch is a device of isolating voltage in order to ensure the safety of the maintenance work of high voltage apparatus and equipment. It is installed by bolts on the mounting rack of electric equipment. The isolating switch is exposed outdoors and always affected by wind, rain, dust and so on, resulting in corrosion, sludge attachment and irregular thread shape of the isolating switch bolt, which influences the smoothness of screwing isolating switch bolts, and causes inconvenient disassembly and assembly. Therefore, it is necessary to regularly clean, and coat them with grease. Through the research and development of the above tool, the author hopes it will provide some assistance in the future production work.

\section{References}

[1] Ou Zhirong. On the Problems in Substation Equipment Maintenance and Repair of Power Supply Companies and the Measures [J]. Technology and Market, 2013 (08).

[2] Wei Sile. The Problems in Substation Equipment Maintenance and Repair and Countermeasures [J]. China High Technology Enterprises, 2014 (03).

[3] Li Zibin. On the Problems in Substation Equipment Maintenance and Repair and Measures [J].Chinese E-Commerce, 2014 (05).

[4] Guo Jing, Ma Zhiying, Lei Qing. Optimizing deign of Cam Mechanism in Spring Operating Mechanism of Vacuum Circuit Breaker II. High-voltage electrical appliances, 2004,40 (3): 420-423.

[5] Lu Jizhi. On the Condition-based Maintenance and Development of Electric Transmission and Transformation Equipment[J ].Yunan Electric Power. 